\title{
Enhanced Thermal Transport Properties of Epoxy Resin Thermal Interface Materials
}

\author{
Jiao Li, ${ }^{1}$ Ping Zhang, ${ }^{1 *}$ Hong He, ${ }^{1}$ Siping Zhai, ${ }^{1}$ Yaoqi Xian, ${ }^{1}$ Wei Ma ${ }^{2}$ and Liyan Wang ${ }^{2}$
}

In this work, multilayer graphene (MLG), graphene oxide (GO) and carbon nanotube (CNT) are studied as fillers in epoxy resin to enhance thermal transport properties of polymer thermal interface material (TIM). The MLG/CNT filler significantly enhances the thermal conductivity of the epoxy matrix material, increasing thermal conductivity by about $553 \%$ at $25 \mathrm{wt} \%$ load. At the same time, theoretical models are used to predict the thermal conductivity of TIM, and the model predictions are in a reasonable agreement with the experimental values. We also analyzed the thermal contact resistance (TCR) at the interface between the experimentally obtained TIM and solid in detail. The TCR measured at a pressure of $0.75 \mathrm{MPa}$ is $42.8 \mathrm{~mm}^{2} \cdot \mathrm{K} / \mathrm{W}$, which was reduced by a factor of $86.7 \%$ compared to the absence of TIMs (The TCR without adding any thermal interface material is $321.8 \mathrm{~mm}^{2} \cdot \mathrm{K} / \mathrm{W}$ ). It is also established that although MLG contributes more to the thermal conductivity of epoxy resin than GO, GO/epoxy composites are superior to MLG/epoxy composites in reducing the total TCR of solid-solid interface. Our results provide a guideline to enhance thermal transport properties of epoxy resin-based carbon nanocomposites as thermal interface materials (TIMs) for various thermal management applications.

Keywords: Graphene; Thermal interface materials; Thermal conductivity (model); Thermal contact resistance; The boundary resistance

Received 30 January 2019, Accepted 27 February 2019

DOI: $10.30919 / \mathrm{esee} 8 \mathrm{c} 222$

\section{Introduction}

Microelectronic systems are moving toward smaller, more powerful and more efficient, inevitably accompanied by increasing power and power density, which will present new challenges for thermal management. ${ }^{1-3}$ It is well known that thermal problems can lead to a series of reactions, such as, equipment failure, performance degradation, security risks, etc. Thus, how to solve the heat dissipation problem more effectively is critical to the performance, longevity and reliability of electronic devices. ${ }^{4}$ However, the solid-solid contact interface is not a complete contact that is macroscopically displayed, butrather peaks and troughs of the surface are interlaced with each other, and the actual contact area is relatively small. Studies have shown that even if the two surfaces are subjected to $10 \mathrm{MPa}$, the actual contact area is only $1 \%$ to $2 \%$ of the nominal contact area, and the rest is filled with air $(0.02$ $\mathrm{W} /(\mathrm{m} \mathrm{K})$ ) with extremely low thermal conductivity, resulting in a high TCR between the interfaces. ${ }^{5}$ Because of the incomplete contact of the solid-solid surface, the thermal energy of the heat source cannot be efficiently transferred to the heat sink. Therefore, it is extremely urgent to improve the heat transfer between the solid-solid interface, which has

${ }^{1}$ School of Mechanical and Electrical Engineering, Guilin University of Electronic Technology, No. 1 Jinji Road, Guilin, Guangxi 541004, China

${ }^{2}$ Science and Technology on Space Physics Laboratory, China Academy of Launch Vehicle Technology, Beijing 100076, China

*E-mail: pingzhang@guet.edu.cn practical application value and far-reaching significance for the development and development of electronic equipment in the future.

Koorosh et al. filled the aluminum nano-coating in the solid-solid interfacial gap, and the results showed that the value of TCR decreases about 38\%, after nanocoating. ${ }^{6}$ Qiu et al. found that adjusting the height of carbon nanotubes as TIMs can improve the interface heat transfer between vertically aligned CNT arrays and heat sinks. ${ }^{7}{ }^{8}$ Wang et al. reduced TCR using a TIM that synthesized aligned carbon nanotubes (CNTs) on both sides of a thin copper foil. ${ }^{9}$ It has been reported that the TCR is reduced by filling the TIM between the solid-solid interfaces. ${ }^{10-12}$ The heat transfer capacity between the rough solid-solid interface can be represented by the total TCR, which is mainly composed of two part: (1) the bulk resistance $\left(R_{B L T}\right)$, (2) the boundary resistance $\left(R_{C_{1} 2}\right) \cdot{ }^{13-15}$ It can be seen that the total TCR comprises contributions from the $R_{B L T}$ of the TIM and $R_{C_{12}}$ from the interfaces between the TIM and solid-solid interface. It is worth noting that extensive research is focused on improving the thermal conductivity of TIMs, while ignoring the reduction of total TCR in practical applications is the ultimate goal. Although $R_{B L T}$ can be reduced by increasing the thermal conductivity of TIMs, it is unclear whether this method can effectively reduce the total TCR. Simultaneously, the effect of $R_{B L T}$ also depends on the bond line thickness of TIM. Additionally, one of the contributions to the total TCR is a non-negligible factor that is the $R_{C_{12}}$. But the factors affecting the thermal resistance of TIMs are many and very complex, such as the viscosity, the bond line thickness, thermal conductivity of the TIM, the pressure load, the surface topography at the contact interface, etc. Therefore, our research focus on reducing the bond line thickness of TIMs and the boundary resistance while maximizing the thermal 
conductivity of the TIM, so as to obtain the minimum total TCR to meet the practical application requirements.

In practical applications, the ideal TIM is required to have high thermal conductivity, and the combination of outstanding electrical insulation, flexibility, easy processing and good design freedom., 16 Polymers are widely used in thermal management due to their excellent properties, such as light weight, high corrosion resistance, flexible processability, excellent electrical insulation properties, mechanical and fatigue resistance. Unfortunately, the inherent low thermal conductivity coefficient of polymers restricts their broader application. Usually, the TIM consists of a thermally conductive filler and a polymer matrix material to overcome the low thermal conductivity of the polymer itself. The most common matrix material is epoxy resin, which has received great commercial and research interests in heat dissipation fields because of its excellent mechanical properties, heat resistance, chemical stability and molding process. Typical thermal conductive fillers, include silver $(\mathrm{Ag}),{ }^{17,18}$ silicon carbide $(\mathrm{SiC}){ }^{19}$ aluminum oxide $\left(\mathrm{Al}_{2} \mathrm{O}_{3}\right)^{20,21}$ aluminum nitride $(\mathrm{AIN}){ }^{22-24}$ and boron nitride $(\mathrm{BN}){ }^{25}$ and so on. Lee et al. studied the thermal conductivity of composites filled with zinc oxide $(\mathrm{ZnO}), \mathrm{SiC}$, or $\mathrm{BN}$, and their thermal conductivity values reached 2.26 , 2.85 , and $2.08 \mathrm{~W} /(\mathrm{m} \mathrm{K})$ respectively at a filler content of $60 \mathrm{vol} \%{ }^{26} \mathrm{Hu}$ et al. prepared epoxy resin/oriented $\mathrm{BN}$ composite material by a facile hot-pressing strategy, and the thermal conductivity of composite was increased from 1 to $5 \mathrm{~W} /(\mathrm{m} \mathrm{K})$ at $50 \mathrm{wt} \%$ loading. ${ }^{27}$ Theoretically, a high thermal conductivity can be obtained by adding high filler loading, but this method in turn will increase the economic cost and damage the mechanical properties, resulting in a large weight of the equipment. Since the discovery of graphene, it has attracted the attention of a large number of researchers. ${ }^{28,29}$ Using carbon materials is considered to be an effective method to achieve a higher thermal conductivity with low filler contents, while reducing problems associated with density and mechanical properties. ${ }^{14,30,31}$

In order to improve the dispersibility of the filler as much as possible, in this study, composite TIM was prepared with GO, MLG and CNT as fillers and epoxy resin as a matrix material through a typical method of solution blending, which the method is relatively simple and easy to realize industrial production. The influences of filler type and content on thermal conductivity of composites were briefly investigated. Using the TCR test system, the $R_{B L T}$ and $R_{C_{12}}$ of TIMs prepared by doping different thermally conductive particles in epoxy resin were investigated. This study provides some guidance for the practical application of TIMs. In addition, we developed a two-step model based on the models of Maxwell model and Nan's model for thermal conductivity of graphene composite.

\section{Experiment section}

\subsection{Material}

The natural flake graphite (100 mesh, $99 \%$ ) was obtained from Nanjing XFNANO Materials Tech Co. Ltd., China. Multilayer graphene (MLG) and carbon nanotube (CNT) were supplied by Suzhou TANFENG graphene Tech Co., Ltd. The epoxy resin and other additives were purchased from Hangzhou WUHUIGANG adhesive Co. Ltd. Concentrated sulfuric acid $\left(\mathrm{H}_{2} \mathrm{SO}_{4}, 95-98 \%\right)$, potassium permanganate $\left(\mathrm{KMnO}_{4}, \geqslant 99.5 \%\right)$, hydrogen peroxide $\left(\mathrm{H}_{2} \mathrm{O}_{2}, 30 \%\right)$, concentrated hydrochloric acid $(\mathrm{HCl}, 36-38 \%)$ and all organic solvents were of analytical grade supplied by XiLong Scientific Co., Ltd. Deionized water with electrical resistivity of $18.2 \mathrm{M} \Omega \cdot \mathrm{cm}^{-1}$ was used in all experiments.

\subsection{Synthesis of Graphene Oxide (GO)}

GO was synthesized using the modified Hummer's method. ${ }^{32-34}$ In the ice water bath environment, $1 \mathrm{~g}$ natural flake graphite was placed into 23 $\mathrm{ml}$ of concentrated sulfuric acid and stirred evenly. $5 \mathrm{~g} \mathrm{KMnO}_{4}$ powder was slowly added to keep the temperature of the reaction lower than $5{ }^{\circ} \mathrm{C}$ and continuously mix for $2 \mathrm{~h}$. Then, the mixture was heated to $35{ }^{\circ} \mathrm{C}$ and stirred for $30 \mathrm{~min}$. An appropriate amount of deionized water was added slowly to the reactor until the temperature of the reaction system is not rising and temperature control below $98{ }^{\circ} \mathrm{C} .20 \mathrm{ml} 30 \% \mathrm{H}_{2} \mathrm{O}_{2}$ was added to mixture and centrifugal removal of supernatant. After, the product was washed several times with $\mathrm{HCl}$ solution and deionized water, respectively, to remove the metallic ions and impurities. The washed sample is sufficiently dried in a vacuum oven at $60{ }^{\circ} \mathrm{C}$ to obtain graphite oxide. The attained graphite oxide was dispersed in deionized water by ultrasonication to exfoliate the graphite oxide to $\mathrm{GO}$, which was dried in a vacuum oven at $60{ }^{\circ} \mathrm{C}$ for $48 \mathrm{~h}$.

\subsection{Preparation of epoxy resin composites}

In this study, the composite material was prepared by solution blending to improve the compatibility of the filler with polymer-based material. The preparation process is graphically shown in Fig. $1.2 \mathrm{~g}$ of GO particles was suspended in an appropriate amount of acetone under ultrasonication at room temperature for $30 \mathrm{~min}$. Simultaneously, $40 \mathrm{~g}$ epoxy resin was mixed with curing agent (1:0.1weight ratio) by vacuum stirring to uniform. The combination of GO and epoxy resin is a critical step in the preparation of TIMs, which can completely eliminate air by controlling the rate of vacuum agitation. In addition, the same process was used to obtain MLG/epoxy and MLG/CNT/epoxy composite TIM.

\subsection{Characterization}

The thermal conductivity of epoxy composite is measured at room temperature using Hot Disk model TPS2500S thermal constants analyzer (Hot Disk AB, Uppsala, Sweden). The analyzer is a thermal conductivity testing technique based on transient plane source method. When the measured sample has a thermal conductivity ranging from $0.005 \mathrm{~W} /(\mathrm{m} \mathrm{K})$ to $500 \mathrm{~W} /(\mathrm{m} \mathrm{K})$, the measurement error is within $3 \%$. The viscosity of epoxy resin composites is measured with a Digital Viscometer (SNB-2) at uniform rate. The TCR is measured using a laboratory-made high-precision TCR test system device that is designed based on the guidelines from ASTM standard D5470-06 and work done by Kempers, ${ }^{35}$ as shown in Fig. $2 .^{15}$ Heat flows one-dimensionally from top to bottom, and the temperature is linearly distributed within each sample but jumps at the interface due to TCR. The TCR of the TIM is expressed as: ${ }^{36}$

$$
\begin{gathered}
R=\frac{T_{b}-T_{c}}{Q} \\
Q=\left(Q_{1}+Q_{2}\right) / 2 \\
Q_{1,2}=-k \cdot A \cdot \frac{d T}{d x}
\end{gathered}
$$

where $T_{b}-T_{c}$ represent the interfaces temperature drop across the interface between two contacting samples, the interface temperature $T_{b}$ and $T_{c}$ can be achieved by extrapolating the located sensors temperature to the contact interfaces, $\mathrm{Q}$ is the average of the heat fluxes $\mathrm{Q}_{1}$ and $\mathrm{Q}_{2}$ in the upper and lower meter-bars, $\mathrm{k}$ is the thermal conductivity of the samples, A is the sectional area, $d T / d x$ is the temperature gradient. The uncertainty of the system is $2 \%$ under the premise of not considering the hardness, roughness and flatness of the contact surface. ${ }^{37}$ It is worth noting that, in order to reduce the experimental error, acetone and alcohol were used to clean the surface of the sample table before the 


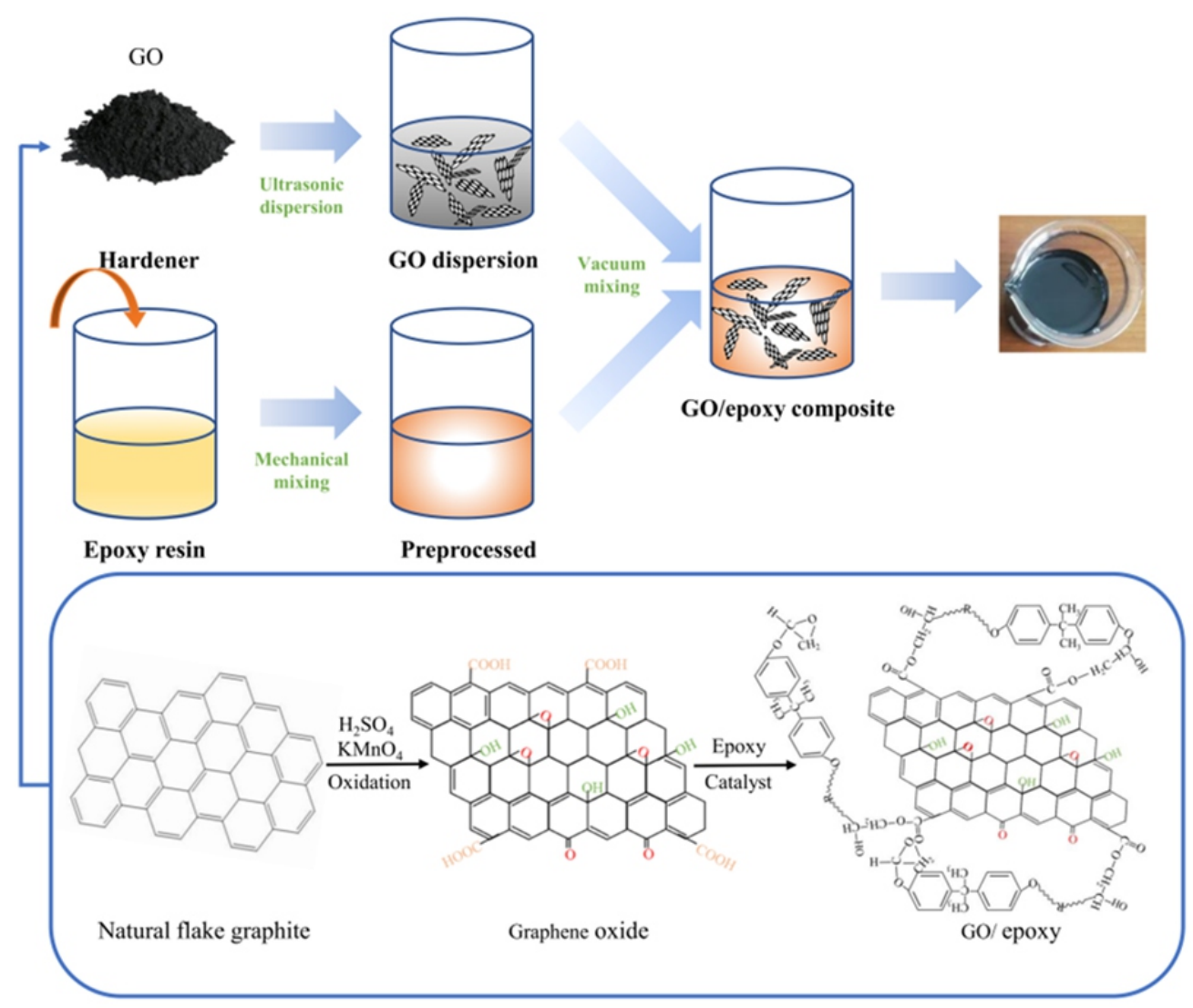

Fig. 1 Schematic diagram of the fabrication procedure of the GO/epoxy composite, and schematic of synthesis.

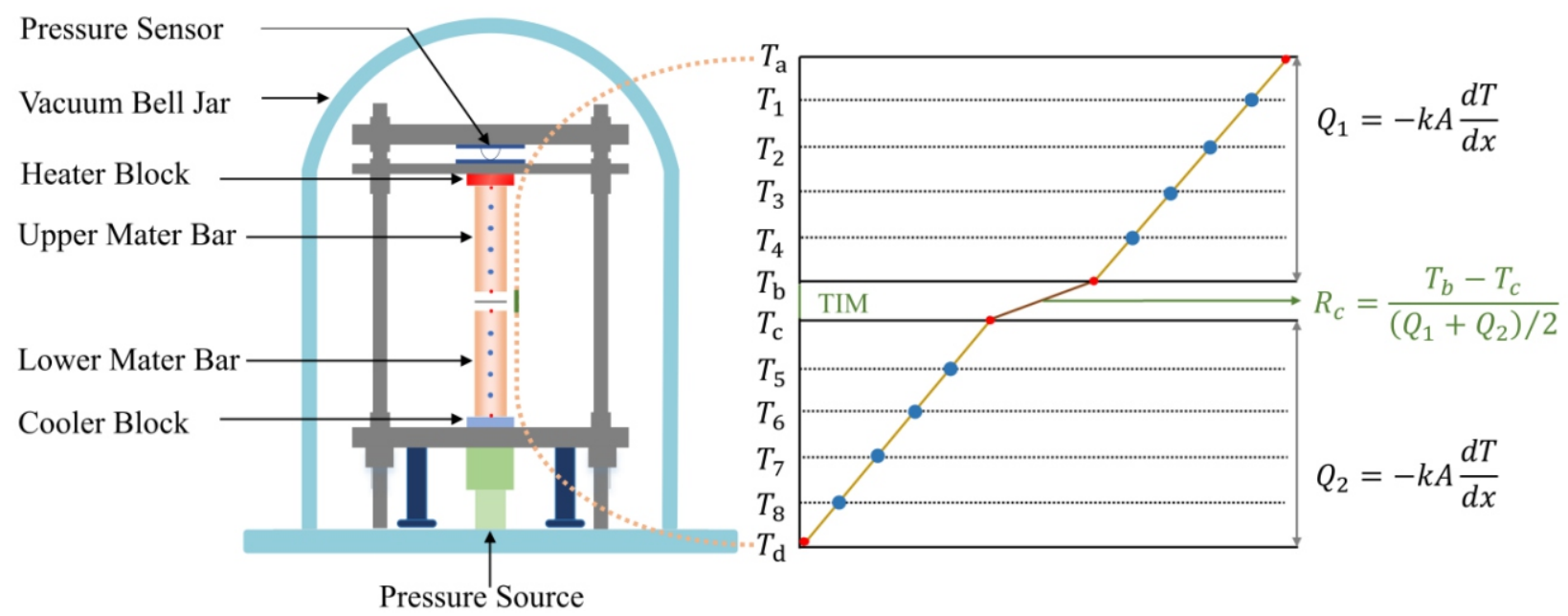

Fig. 2 The illustration shows the measuring principle of TCR after adding TIM. ${ }^{15}$ 
test. Meanwhile, in order to avoid the influence of sample thickness, the TIM was applied to the contact surface by means of a 500 mesh wire mesh.

\section{Results and discussion}

3.1 Effect of viscosity on composite materials

The efficient viscosity of the TIM reflects its flow properties, which play a vital role for the processing technology and performance of

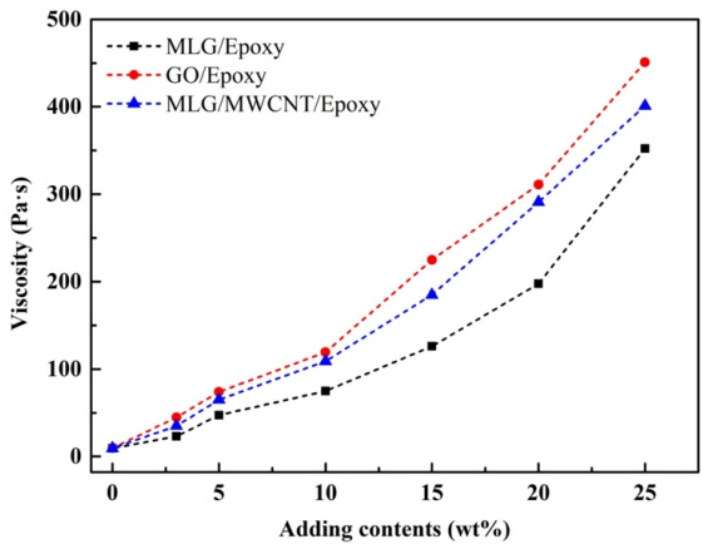

Fig. 3 Viscosity of MLG/epoxy, GO/epoxy and MLG/CNT/epoxy at room temperature. materials. Fig. 3 shows the relationship between the viscosity of the epoxy resin interface material and the filler content. As the filler content increases, the viscosity of the epoxy resin composite gradually increases. But excessive viscosity makes the composites very difficult to process, limiting their real-world applications as TIMs. ${ }^{38}$ Comparing the three different fillers, GO has the least effect on the viscosity of the polymer, and MLG has the most obvious effect on the viscosity of the polymer. This is because that the adsorption capacity between the GO and the epoxy resin is strong, so that the viscosity of the composite material is increased.

\subsection{Thermal conductivity of composites}

Fig. 4(a) shows the thermal conductivity of epoxy resin composites with different filler loading. One can see from diagram that each type of filler structure results in a dramatic enhancement of thermal conductivity of the composites in comparison with the epoxy resin, and the effect of MLG/CNT binary mixed filler is most prominent. It is noteworthy that the thermal conductivity increases rapidly after $15 \mathrm{wt} \%$. This phenomenon is mainly due to the matrix materials plays a major role at the beginning, and as the filler content increases, the heat conduction path formed between particles plays a major role, as shown in Fig. 4(c). When the content of MLG, GO and MLG/CNT is $25 \%$, the thermal conductivity of the prepared TIM is increased by $6.0,4.0$, and 6.5 times compared with the epoxy resin matrix material, respectively. One of the main reasons for these results is that the CNT can interconnect the layers of the MLG sheets to a certain extent to form a

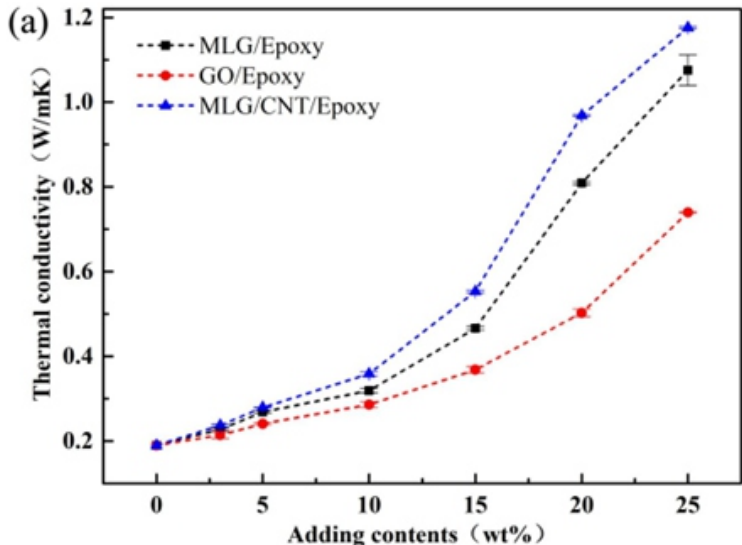

(c)

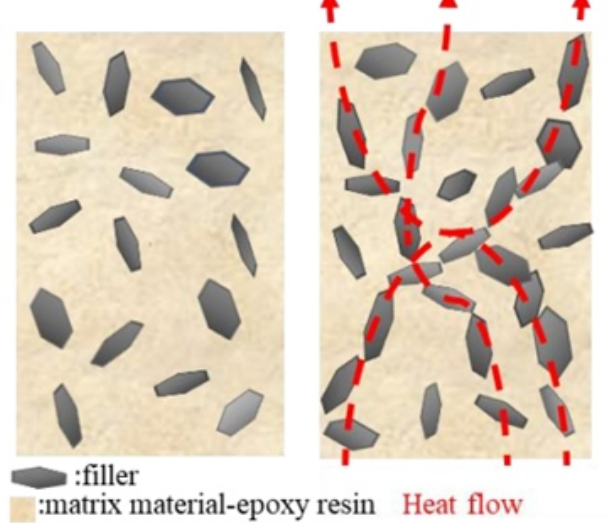

(b)

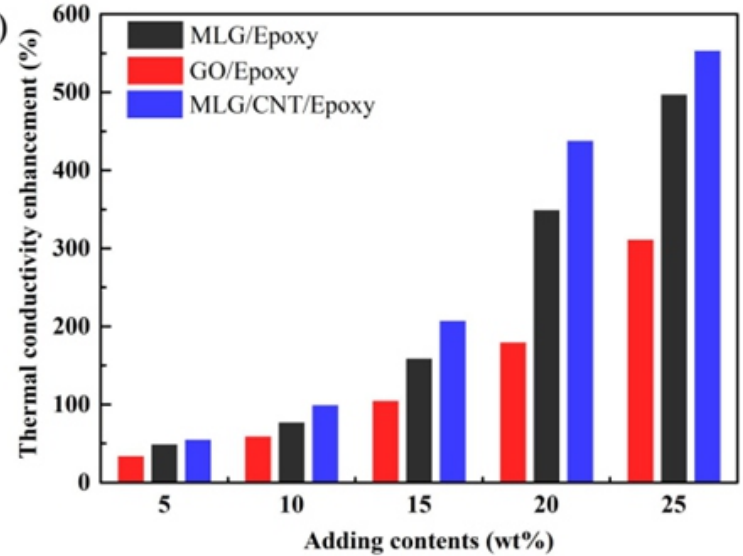

(d)

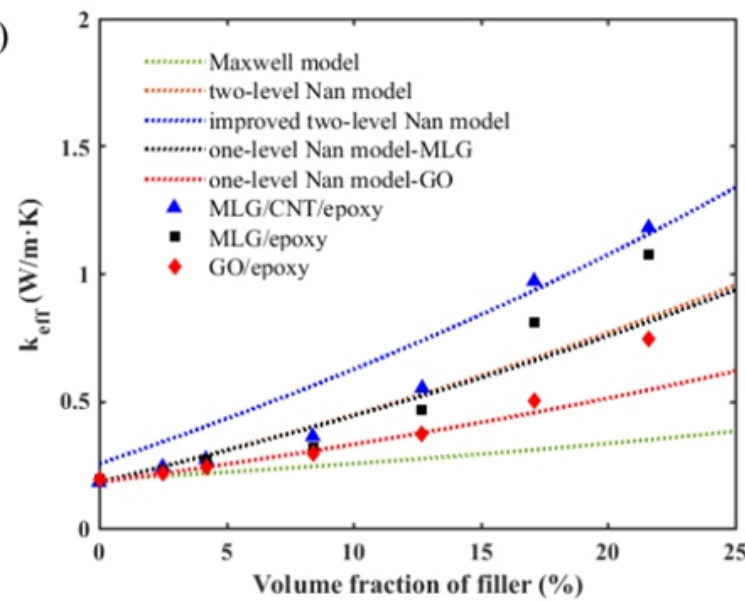

Fig. 4 Thermal properties of epoxy resin composites and their comparison composites. (a) Thermal conductivity of GO/epoxy, MLG/epoxy, and MLG/CNT/epoxy composites as a function of weight fraction. (b) Composite material thermal conductivity enhancement efficiency. (c) Respective heat dissipation model of prepared composites. (d) The prediction of several models with experiment data for polymer composites. 
three-dimensional heat conduction path, which promotes the heat transfer and makes with the thermal conductivity higher. ${ }^{39}$ GO is obtained by treating graphite with strong acid. The surface covalently bonds many oxygen-containing groups such as carboxyl groups and hydroxyl groups, resulting in a significant decrease in its own thermal conductivity, which limits its contribution to the thermal conductivity of composites. To further elucidate the extent of improvement, a parameter $\eta$ was introduced, which is defined as:

$$
\eta=\frac{K_{c}-K_{m}}{K_{m}} \times 100 \%
$$

where $K_{C}$ and $K_{m}$ represent the thermal conductivity of the composites and pure epoxy matrix, respectively. Fig. 4 (b) shows that $\eta$ increases with increasing filler loading same as the trend of thermal conductivity growth, the $\eta$ value increases rapidly after the filler loading is $15 \mathrm{wt} \%$. At 25 wt \% loading, the $\eta$ values of GO/epoxy, MLG/epoxy and MLG/CNT/epoxy dramatically up to $311 \%, 497 \%$ and $553 \%$, respectively.

In order to predict the thermal conductivity of composites, many theoretical models have been reported. The original model is the Maxwell model, ${ }^{40}$ but it is so simple that it is difficult to adapt to the complexities of particle-filled composites. Subsequently, scholars have proposed many models to research the thermal conductivity of particlefilled composites. The noticeable study was by Nan et al., ${ }^{41}$ who adopted the multiple scattering theory to propose a more general model. The Nan's model considers the effects of the properties of the matrix and particle, particle size, Kapitza resistance and orientation distribution of the particles.

In this study, based on the Nan model, we present a two-level Nan's model to consider hybrid particles. The first level is performed with only the MLG. The thermal conductivity of the MLG/epoxy composites is calculated by:

$$
\begin{gathered}
k_{e f f}^{M L G}=k_{m} \frac{3+f_{p}^{M L G}\left[2 \beta_{x}^{M L G}\left(1-L_{x}^{M L G}\right)+\beta_{z}^{M L G}\left(1-L_{z}^{M L G}\right)\right]}{3-f_{p}^{M L G}\left[2 \beta_{x}^{M L G} L_{x}^{M L G}+\beta_{z}^{M L G} L_{z}^{M L G}\right]} \\
\beta_{i}^{M L G}=\frac{k_{p i}^{M L G}-k_{m}}{k_{m}+L_{i}^{M L G}\left(k_{p i}^{M L G}-k_{m}\right)}, i=x, y \text { and } z
\end{gathered}
$$

where the $L_{i}^{M L G}(i=\mathrm{x}, \mathrm{y}$ and $\mathrm{z})$ is the depolarization factor of the MLG along the $i$-axis $(i=\mathrm{x}, \mathrm{y}$ and $\mathrm{z}), k_{p i}^{M L G}$ is the effective thermal conductivity of MLG along the $i$-axis $(i=\mathrm{x}, \mathrm{y}$ and $\mathrm{z})$, and the $k_{p}^{M L G}$ is the volume fraction of MLGs.

The thermal conductivity of MLG/CNT/epoxy composites is calculated by assuming that the CNT is embedded in an effective matrix with a thermal conductivity of $k_{e f f}^{M L G}$. Using the model by Nan et al., the thermal conductivity of the MLG/CNT/epoxy composites is given by:

$$
\begin{gathered}
k_{e f f}=k_{e f f}^{M L G} \frac{3+f_{p}^{C N T}\left[2 \beta_{x}^{C N T}\left(1-L_{x}^{C N T}\right)+\beta_{z}^{C N T}\left(1-L_{z}^{C N T}\right)\right]}{3-f_{p}^{C N T}\left[2 \beta_{x}^{C N T} L_{x}^{C N T}+\beta_{z}^{C N T} L_{z}^{C N T}\right]} \\
\beta_{i}^{C N T}=\frac{k_{p i}^{C N T}-k_{e f f}^{M L G}}{k_{e f f}^{M L G}+L_{i}^{C N T}\left(k_{p i}^{C N T}-k_{e f f}^{M L G}\right)}, i=x, y \text { and } z
\end{gathered}
$$

where the $L_{i}^{C N T}(i=\mathrm{x}, \mathrm{y}$ and $\mathrm{z})$ is the depolarization factor of the CNT along the $i$-axis ( $i=\mathrm{x}, \mathrm{y}$ and $\mathrm{z}), k_{p i}^{C N T}$ is the effective thermal conductivity of CNT along the $i$-axis ( $i=\mathrm{x}, \mathrm{y}$ and $\mathrm{z}$ ), and the $f_{p}^{C N T}$ is the volume fraction of CNTs. And $L_{\mathrm{x}}$ and $L_{\mathrm{z}}$ are the depolarization factors related to the nanoparticle shape ${ }^{41}$ :

$$
\begin{gathered}
L_{x}=\left\{\begin{array}{cc}
\frac{p^{2}}{2\left(p^{2}-1\right)}+\frac{p}{2\left(1-p^{2}\right)^{1.5}} \cdot \frac{1}{\cos p}, & \text { for } p<1 \\
\frac{p^{2}}{2\left(p^{2}-1\right)}-\frac{p}{2\left(p^{2}-1\right)^{1.5}} \cdot \frac{1}{\cosh p}, & \text { for } p>1
\end{array}\right. \\
L_{z}=1-2 L_{x}
\end{gathered}
$$

where $p=a_{3} / a_{1}$ is the aspect ratio of the particle, and $p<1$ and $p>1$ are for a MLG $\left(a_{1}=a_{2}>a_{3}\right)$ and a CNT $\left(a_{1}=a_{2}<a_{3}\right)$, respectively.

The theoretical thermal conductivity values calculated by onelevel Nan model and two-level Nan model were shown in Fig. 4(d), and the value calculated by the original Maxwell model was also presented for comparison. It is noted that compared with the original Maxwell equation, both the one-level Nan model and improved two-level Nan model show a better match with the experimental results. As can be seen from Fig. 4(d), experiment values for both MLG/epoxy, GO/epoxy and MLG/CNT/epoxy compositesare in good agreementwith those predicted by the one-level Nan model and two-level Nan model when the fraction of nanofillers was less than 0.05 vol\%. However, as the fraction increases from 0.05 to $0.15 \mathrm{vol} \%$, the curve of the theoretical value gradually deviates from that of experimental value and is slightly higher than it. This discrepancy may be due to the fact that the theoretical models fail to fully consider the interfacial thermal resistance in composites, mainly the interfacial thermal resistance between nanofillers and that caused by the voids in composites. When the fraction exceeds $0.15 \mathrm{vol} \%$, the experiment values increase rapidly, and the experiment values of both MLG/epoxy, GO/epoxy and MLG/CNT/epoxy composites are larger than theoretical values at the weight fraction of 0.2. This result can be attributed to the fact that presented models do not consider the synergistic effect of hybrid particles, which has been discussed by Yu et al. in detail. ${ }^{42}$ In order to consider the synergistic effect of hybrid particles, we improve the twolevel Nan's model by adding a synergistic factor $\xi$, the Eq. (3c) expands as follows: ${ }^{42}$

$$
k_{e f f}=\xi \cdot k_{e f f}^{M L G} \frac{3+f_{p}^{C N T}\left[2 \beta_{x}^{C N T}\left(1-L_{x}^{C N T}\right)+\beta_{z}^{C N T}\left(1-L_{z}^{C N T}\right)\right]}{3-f_{p}^{C N T}\left[2 \beta_{x}^{C N T} L_{x}^{C N T}+\beta_{z}^{C N T} L_{z}^{C N T}\right]}
$$

where the values of $\xi$ is appropriately set to 1.3 . The values calculated by the improved two-level Nan's model were shown in Fig. 4(d), which are in good agreement with the experimental values.

\subsection{Thermal contact resistance of composite materials}

Fig. 5(a) clearly shows the test method for the TCR of composite TIMs as a function of pressure. The test results evidently show in Fig. 5 (b), which indicated that the interface thermal resistance decreases with increasing pressure at a filler of $25 \mathrm{wt} \%$ loading. When the solid-solid interface is filled with no TIM, a very high TCR value appears (1413 $\left.\mathrm{mm}^{2} \cdot \mathrm{K} / \mathrm{W}\right)$. We attribute this to that the inter-interface filled with air, which is very low in thermal conductivity. Furthermore, the TCR is 193 $\mathrm{mm}^{2} \cdot \mathrm{K} / \mathrm{W}$ when the interface is filled with $\mathrm{GO} /$ epoxy composite, which is 7.3 times lower than that without the interface material. After application of MGL/epoxy composite material, the TCR is reduced to $219 \mathrm{~mm}^{2} \cdot \mathrm{K} / \mathrm{W}$, which is reduced by 6.5 times. MGL/CNT/epoxy composites are used between interfaces to reduce contact resistance to $166 \mathrm{~mm}^{2} \cdot \mathrm{K} / \mathrm{W}$, which is 8.5 times lower. The most important information about the reduction of TCR of the GO/epoxy composite under the same loading can be obtained from the experimental results. Combined with the results of thermal conductivity measurement (Fig. 4 (a)), the thermal conductivity of MGL/epoxy composites is generally higher than that of GO/epoxy composites, but the degree of thermal 
resistance reduction is lower than that of $\mathrm{GO} /$ epoxy resin composites. This may be due to the higher viscosity of the GO/ epoxy system than the MGL/epoxy system, making the contact with the solid-solid interface more complete. Therefore, thermal conductivity is not the only factor affecting TCR, but also needs to consider other factors, such as viscosity, the roughness of the contact surface, etc. Further analysis shows that the pressure of $0.5 \mathrm{MPa}$ is a demarcation point, before which the TCR is rapidly reduced, after which the TCR is gently reduced. This is because as the pressure increases, the contact area between the TIM and the solid interface gradually increases, resulting in a rapid decrease of TCR, and when the pressure reaches a certain value, the TIM completely contacts the solid interface, which leads to the TCR tends to develop steadily. Therefore, in practical applications, the effect of pressure on the thermal properties of TIM enhancement devices is also limited. So, we need a more specific analysis of the TCR of the composite at a pressure of $0.5 \mathrm{MPa}$. Under the test condition of 0.5 $\mathrm{MPa}$, the bond line thickness of the TIM coated between the heat flow meters is $11 \pm 0.5 \mu \mathrm{m}$ by TCR test system (the BLT $=11 \pm 0.5 \mu \mathrm{m}$ ).

Fig. 6 shows the total TCR, $\mathrm{R}_{\mathrm{BLT}}$ and $\mathrm{R}_{\mathrm{Cl}}+\mathrm{R}_{\mathrm{C} 2}$ of epoxy composites added with different proportions of carbon filler at $0.5 \mathrm{MPa}$ pressure loading. It can be seen from the figure that the prominent decrease of the total TCR is observed in the epoxy composites filled with fillers. A special trend was found that composite $\mathrm{R}_{\mathrm{Cl}}+\mathrm{R}_{\mathrm{C} 2}$ suddenly increased as filling 15\% MLG. But, the total TCR decreases from 116.2 to 80.6 $\mathrm{mm}^{2} \cdot \mathrm{K} / \mathrm{W}$ with the MLG content increases, and the minimum value is obtained when the MLG fills $25 \mathrm{wt} \%$. This is because that when the adding fraction is large, the viscosity of the epoxy resin composite TIM is increased, and the fluidity is disappointing, so that the bonding performance with the solid-solid surface is deteriorated, resulting in a sudden increase in the value of $\mathrm{R}_{\mathrm{C} 1}+\mathrm{R}_{\mathrm{C} 2}$. Another cause of unsatisfactory results is the large interfacial thermal resistance between the matrix material and the filler. However, it indicated that the total TCR tends to decrease as the proportion of MLG increases. This was attributed to the high thermal conductivity of the MLG/epoxy resin composite such that the volumetric thermal resistance is relatively low, which ultimately promotes a reduction in total TCR. The results in Fig. 6 show that $R_{\text {BLT }}$ shows a linear downward trend with the increase of $\mathrm{GO}$ content. $\mathrm{R}_{\mathrm{BLT}}$ is $51.3 \mathrm{~mm}^{2} \cdot \mathrm{K} / \mathrm{W}$ as the addition of $3 \mathrm{wt} \%$, accounting for $48 \%$ of the total TCR. When the added fraction is $25 \mathrm{wt} \%$, the thermal resistance of the volume is about $14.9 \mathrm{~mm}^{2} \cdot \mathrm{K} / \mathrm{W}$, which accounts for $23 \%$ of the total TCR. Further analysis shows that the $\mathrm{R}_{\mathrm{C} 1}+\mathrm{R}_{\mathrm{C} 2}$ of $\mathrm{GO} /$ epoxy composite has been maintained at around 50 with no significant change with the increase of GO filler content. Besides, GO/epoxy composites always exhibit low $\mathrm{R}_{\mathrm{C} 1}+\mathrm{R}_{\mathrm{C} 2}$ values compared to MLG/epoxy composites. We can conclude that although MLG contributes more to the thermal conductivity of epoxy resin than GO, GO/epoxy composites are superior to MLG/epoxy composites in reducing the total TCR of solid-solid interface. So, GO/epoxy composites have more practical significance than MLG/epoxy composites. For MLG/CNT/epoxy composites, the total TCR of the composite decreases from 96.8 to 53.8 $\mathrm{mm}^{2} \mathrm{~K} / \mathrm{W}$ as the filled proportions of MLG/CNT increase. The ratio of $\mathrm{R}_{\mathrm{Cl}}+\mathrm{R}_{\mathrm{C} 2}$ to the total TCR increases from $52 \%$ to $82 \%$ with increasing the filled MLG/CNT content. Obviously, the addition of MLG/CNT plays a considerable role in the change of $\mathrm{R}_{\mathrm{Cl}}+\mathrm{R}_{\mathrm{C} 2}$ between the contact substrate and the composite, which results in an unsatisfactory total TCR effect. However, we obtained a satisfactory $R_{B L T}$ due to the increase in the MLG/CNT filler to increase the thermal conductivity of the composite. In summary, MLG/CNT is the most effective filler to reduce total TCR, followed by GO and MLG.

\section{Conclusions}

In this study, we successfully prepared epoxy resin composites by solution blending method. It was found that that the carbon material significantly enhances the thermal conductivity of the epoxy resin matrix material, and the reinforcing effect of the MLG/CNT filler is optimal, followed by MLG and GO. It was also established that the presence of TIMs always significantly reduces the TCR, but the effect of pressure on the thermal properties of the TIM between the solid-solid interface is limited. The surprising finding is that, although GO/epoxy composites have low thermal conductivity, they are superior to MLG/epoxy composites with high thermal conductivity in reducing the total TCR at the solid-solid interface. In addition, a two-step theoretical model for thermal conductivity of graphene composite is developed by modifying the models of Maxwell model and Nan's model, and the model predictions are in reasonable agreement with the experimental values. The results obtained provide a valuable technical reference for using thermal interface materials to improve equipment heat problems in practical applications.

\section{Acknowledgements}

The author(s) disclosed receipt of the following financial support for the research, authorship, and/or publication of this article: The authors acknowledge the financial support provided by National Natural

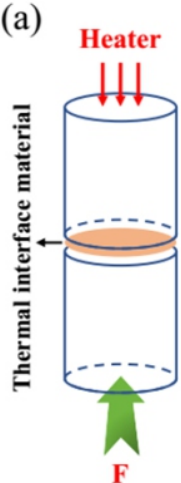

(b)

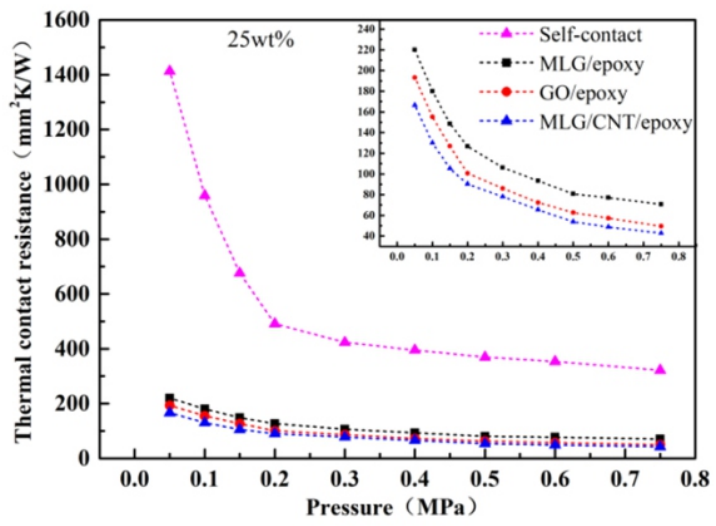

Fig. 5 (a) TCR measurement schematic. (b) Functional relationship between pressure and TCR.

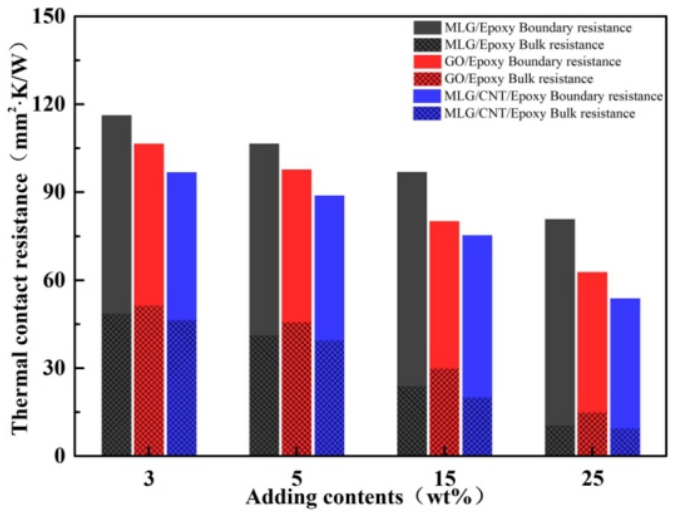

Fig. 6 Total TCR, $\mathrm{R}_{\mathrm{BLT}}$ and $\mathrm{R}_{\mathrm{Cl}}+\mathrm{R}_{\mathrm{C} 2}$ of the epoxy composite at $0.5 \mathrm{MPa}$ pressure loading (the $\mathrm{BLT}=11 \pm$ $0.5 \mu \mathrm{m})$. 
Science Foundation of China (Project No. 51506033), Innovation Project of GUET Graduate Education (Grant No. 2018YJCX03), the Guangxi Colleges and Universities Program of Innovative Research Team and Outstanding Talent, Guangxi Natural Science Foundation (Grant No. 2017JJA160108), and GUET Excellent Graduate Thesis Program (Grant No. 16YJPYSS03).

\section{References}

1. M. A. Vadivelu, C. R. Kumar and G. M. Joshi, Compos. Interface, 2016, 23, $1-26$.

2. X. C. Tong, Advanced Materials for Thermal Management of Electronic Packaging, Springer New York, 2011.

3. A. L. Moore and L. Shi, Mater. Today, 2014, 17, 163-174.

4. Y. Zhou, F. Liu and H. Wang, Polym. Composite., 2015, 38, 803-813.

5. M. Yovanovich and E. Marotta, Heat Transfer Handbook, 2003, 1, 261-394.

6. K. Goodarzi, S. R. Ramezani and S. Hajati, Appl. Therm. Eng., 2014, 70, 641-646.

7. L. Qiu, P. Guo, Q. Kong, C. W. Tan, K. Liang, J. Wei, J. N. Tey, Y. Feng, X. Zhang and B. K. Tay, Carbon, 2019, 145, 725-733.

8. L. Qiu, K. Scheider, S. A. Radwan, L.S. Larkin, C. B. Saltonstall, Y. Feng, X. Zhang and P. M. Norris, Carbon, 2017, 120, 128-136.

9. H. Wang, J. Y. Feng, X. J. Hu and K. M. Ng, Chem. Eng. Sci., 2010, 65, 1101-1108.

10. D. Kang, H. Ko and J. Koo, ACS Appl. Mater. Inter, 2018, 10, 35557-35562.

11. A. J. Mcnamara, Y. Joshi and Z. M. Zhang, Int. J. Therm. Sci., 2012, 62, 2-1 .

12. M. T. Barako, S. G. Isaacson, F. Lian, E. Pop, R. H. Dauskardt, K. E. Goodson and J. Tice, ACS Appl. Mater. Inter, 2017, 9, 42067-42074.

13. R. S. Prasher and J. C. Matayabas, IEEE T. Compon. Pack. T., 2004, 27, 702-709.

14. K. M. Shahil and A. A. Balandin, Nano Lett., 2012, 12, 861-867.

15. P. Zhang, Q. Li and Y. M. Xuan, Compos. Part A-Appl. S., 2014, 57, 1-7.

16. J. Yang, Y. Ye, X. Li, X. Lü and R. Chen, Compos. Sci. Technol., 2018, 164, 187-194.

17. K. Pashayi, H. R. Fard, F. Lai, S. Iruvanti, J. Plawsky and T. Borca-Tasciuc, J. Appl. Phys., 2012, 111, 2474.

18. F. Wang, X. Zeng, Y. Yao, R. Sun, J. Xu and C. P. Wong, Sci. Rep., 2016, 6, 19394.

19. D. Shen, Z. Zhan, Z. Liu, Y. Cao, L. Zhou, Y. Liu, W. Dai, K. Nishimura, C. Li and C. T. Lin, Sci. Rep., 2017, 7, 2606.

20. Y. X. Fu, Z. X. He, D. C. Mo and S. S. Lu, Appl. Therm. Eng., 2014, 66, 493-498.
21. W. Yu, H. Xie, L. Yin, J. Zhao, L. Xia and L. Chen, Int. J. Therm. Sci., 2015, 91, 76-82.

22. Y. Xu, D. D. L. Chung and C. Mroz, Compos. Part A-Appl. S., 2001, 32, 1749-1757.

23. C. Yu, J. Zhang, Z. Li, W. Tian, L. Wang, J. Luo, Q. Li, X. Fan and Y. Yao, Compos. Part A-Appl. S., 2017, 98, 25-31.

24. W. Yuan, Q. Xiao, L. Li and T. Xu, Appl. Therm. Eng., 2016, 106, 10671074 .

25. J. M. Hutchinson, F. Roman and A. Folch, Polymers-Basel, 2018, 10, 340.

26. B. Lee and B. Lee, Express Polym. Lett., 2008, 2, 357-363.

27. J. Hu, Y. Huang, X. Zeng, Q. Li, L. Ren, R. Sun, J. B. Xu and C. P. Wong, Compos. Sci. Technol., 2018, 160, 127-137.

28. C. Q. Liu, M. Chen, W. Yu and Y. He, ES Energy Environ., 2018, 2, 31-42.

29. L. Qiu, P. Guo, H. Y. Zou, Y. H. Feng, X. X. Zhang, S. Pervaiz and D. S. Wen, ES Energy Environ., 2018, 2, 66-72.

30. W. L. Song, W. Wang, L. M. Veca, C. Y. Kong, M. S. Cao, P. Wang, M. J. Meziani, H. Qian, G. E. Lecroy and L. Cao, J. Mater. Chem., 2012, 22, 17133-17139.

31. L. Qiu, P. Guo, X. Yang, Y. Ouyang, Y. Feng, X. Zhang, J. Zhao, X. Zhang and Q. Li, Carbon, 2019, 145, 650-657.

32. W. S. H. Jr and R. E. Offeman, J. Am. Chem. Soc, 1958, 80, 1339.

33. I. K. Nina, J. O. Patricia, R. M. Benjamin, E. M. Thomas, A. C. Sergey, E.V.B. And and A.D. Gorchinskiy, Chem. Mater., 1999, 11, 771-778.

34. P. Kumar, F. Shahzad, S. Yu, S. M. Hong, Y. H. Kim and M. K. Chong, Carbon, 2015, 94, 494-500.

35. R. Kempers, P. Kolodner, A. Lyons and A.J. Robinson, Rev. Sci. Instrum., 2009, 80, 1379-1386.

36. P. Zhang, Y. Xuan and Q. Li, Exp Therm. Fluid. Sci., 2014, 54, 204-211.

37. P. Zhang, B. Shi, Y. M. Xuan and Q. Li, Meas. Sci. Technol., 2013, 24, 95004

38. P. Ravis S, K. Paul and S. James, J. Electron. Packaging, 2003, 125, 386391.

39. H. Im and J. Kim, Carbon, 2012, 50, 5429-5440.

40. J. C. M. Garnett, 1904, 203, 385-420.

41. C. W. Nan, R. Birringer, D. R. Clarke and H. Gleiter, J. Appl. Phys., 1997, 81, 6692-6699.

42. W. Yu, H. Xie, L. Yin, J. Zhao, L. Xia and L. Chen, Int. J. Therm. Sci., 2015, 91, 76-82.

Publisher's Note Engineered Science Publisher remains neutral with regard to jurisdictional claims in published maps and institutional affiliations. 